\title{
APERTURA INTERNAZIONALE E CAPITALE TERRITORIALE: GLI INVESTIMENTI ESTERI COME NUOVO FATTORE DI CRESCITA DEI DISTRETTI INDUSTRIALI
}

Jel Classification: F23, L25,J24, R12

\author{
di Giancarlo Corò, Mariachiara Barzotto e Ilaria Mariotti
}

\begin{abstract}
$\overline{\text { L'articolo esplora gli effetti della presenza multinazionale nei distretti industriali veneti, }}$ con particolare riferimento ai profili professionali degli occupati. È stata condotta un'analisi controfattuale per confrontare la composizione occupazionale delle imprese distrettuali a controllo italiano (Uni) con quella delle affiliate italiane delle imprese multinazionali estere (Imne) localizzate nei 28 distretti veneti all'anno 2014. Lo studio si avvale di una nuova base dati che comprende: (i) le partecipazioni di affiliate estere in Italia (Reprint-Ice); (ii) i bilanci delle imprese distrettuali, sia Uni che Imne (Aida-Bureau van Dijk); (iii) le informazioni sulla composizione occupazionale delle imprese (Sistema Informativo Lavoro Veneto - Silv). Dall'analisi controfattuale emerge che le imprese affiliate di multinazionali estere tendono ad assumere più personale qualificato e pagare salari più alti, coerentemente con la necessità di attirare lavoratori esperti per recuperare lo svantaggio delle asimmetrie informative.
\end{abstract}

Parole chiave: distretti industriali, imprese multinazionali, investimenti diretti esteri, forza lavoro qualificata

\section{INTERNATIONAL OPENNESS AND TERRITORIAL CAPITAL: FDIS AS NEW GROWTH FACTOR OF INDUSTRIAL DISTRICTS}

The paper explores the effects of inward foreign direct investments (FDIs) on the Veneto' industrial districts, with a focus on the skill composition. A counterfactual analysis has been developed to compare the employment composition of Uninational District Firms (Uni) to the one of inward FDIs located in the 28 industrial districts in 2014. The paper adopts a novel database consisting of: (i) inward FDIs in Italy (Reprint-Ice); (ii) district firms' balance sheets (Aida), both Uni and inward FDIs; (iii) firms' employment composition (Sistema Informativo Lavoro Veneto - Silv). The counterfactual analysis shows that inward FDIs hire a larger share of high skilled workers. Foreign multinational enterprises, indeed, pay higher wages to attract more skilled workers and to cope with information asymmetry.

Key words: Industrial Districts, Multinational Firms, Inward FDIs, Skilled Labour Force 


\section{Gli investimenti esteri come fattore di innovazione}

La tipologia di imprese che popolano il tessuto produttivo dei distretti industriali si è profondamente modificata negli ultimi decenni. In passato la divisione del lavoro in diverse fasi produttive vedeva all'opera numerose piccole e medie imprese che alimentavano una vivace dinamica competitiva all'interno della rete di fornitura. Pur con alcune varianti, l'economia dei distretti operava in base a due principi apparentemente contrapposti. Da un lato, la concorrenza orizzontale e verticale fra imprese creava un insieme di interazioni fra agenti economici attorno a una famiglia di artefatti produttivi, con il risultato di incentivare l'efficienza e generare, attraverso spin-off e scambi informativi per lo più informali, processi continui di creazione e diffusione dell'innovazione. Dall'altro lato, la concentrazione di imprese specializzate su una filiera produttiva funzionava come potente fattore di cooperazione implicita, favorendo la formazione di bacini professionali e lo sviluppo di mercati particolari di fornitura e servizi dedicati. Anche le istituzioni locali - in particolare quelle educative, associative e del credito - risentivano di queste dinamiche, contribuendo a regolare la concorrenza e adattando l'offerta in base a obiettivi condivisi di crescita professionale e sviluppo industriale. All'interno dei distretti si è così andato accumulando un "capitale territoriale" che ha contribuito in misura rilevante ad accrescere la competitività delle imprese sui mercati internazionali (Barzotto, Corò and Volpe, 2016).

Tuttavia, dalla seconda metà degli anni Novanta il funzionamento di questo meccanismo in gran parte spontaneo di generazione di capitale territoriale ha mostrato segnali sempre più evidenti di difficoltà. Le ragioni, più volte richiamate (Corò e Micelli, 2006; Rullani, 2015; Giunta e Rossi, 2017) sono riassumibili nell'irrompere dell'economia digitale e nell'accelerazione dei processi di globalizzazione, di fronte ai quali le imprese distrettuali hanno reagito in modo eterogeneo, rompendo l'equilibrio competitivo che si era creato nei precedenti decenni.

In particolare, le imprese leader hanno esteso le reti di fornitura all'esterno del distretto, sfruttando le nuove aree di offerta di lavoro a basso costo rese accessibili grazie alle nuove tecnologie, a sistemi logistici avanzati, oltre che da un nuovo scenario geopolitico. Per le imprese italiane un impulso verso la delocalizzazione produttiva è stata impresso anche dal nuovo regime monetario dell'Euro, la cui forza ha reso molto più conveniente la creazione o l'acquisizione di filiali all'estero tramite investimenti diretti, oppure anche la più semplice partecipazione a supply chain globali (Corò e Volpe, 2003; De Marchi and Grandinetti, 2014). 
I processi di offshoring hanno avuto effetti economici differenziati nel tempo. Se in una prima fase è prevalsa la convenienza delle imprese alla riduzione dei costi operativi senza incidere in misura rilevante sui prezzi finali, dunque con una crescita della redditività, in un secondo momento si è tuttavia reso evidente il fenomeno di erosione del capitale territoriale dei distretti, con impatti divenuti vieppiù significativi sulla qualità dei prodotti e sulle capacità di innovazione (Pisano and Shih, 2012; Buciuni and Pisano, 2015). Nella misura in cui la creazione di economie esterne distrettuali - in particolare la formazione di competenze distintive - è stato un fenomeno in gran parte spontaneo, generato come sotto-prodotto di processi industriali localizzati, lo spostamento di tali processi oltre i confini distrettuali ha fatalmente indebolito gli industrial commons su cui facevano leva anche le imprese leader dei distretti. I profondi cambiamenti che diversi distretti italiani mettono in luce ancora prima della crisi del 2008 confermano che il vecchio equilibrio fra competizione ben temperata e cooperazione spontanea non funziona più (Guelpa e Micelli, 2007).

L'attenzione verso i processi di offshoring e la creazione di catene globali del valore da parte delle imprese leader dei distretti ha tuttavia fatto perdere di vista un fenomeno non meno importante che contribuisce ad accrescere la complessità e la capacità di tenuta dell'economia locale: gli investimenti esteri in entrata da parte di imprese multinazionali. In questo modo, nell'economia dei distretti entra in gioco un nuovo attore, che in realtà contribuisce a quel processo di riproduzione del capitale territoriale indebolito dalle strategie di offshoring delle imprese locali. La presenza di imprese multinazionali (Imn) svolge infatti un ruolo chiave nel trasferimento e nella diffusione di conoscenza, all'interno o all'esterno del distretto (Morrison, 2008).

Il punto di partenza è che le imprese multinazionali hanno alcune caratteristiche economiche che le rendono importanti fattori di modernizzazione tecnica, produttiva e manageriale (Castellani and Zanfei, 2006). In particolare, come evidenziato ancora tempo fa da Dunning (1993), l'esistenza stessa di un'organizzazione multinazionale è l'espressione di economie di common governance, intese come insieme di vantaggi creati dalla distribuzione delle attività produttive in diverse aree geografiche, alla ricerca di una più efficiente combinazione fattoriale. Oltre all'importanza delle economie di scala, che abilita l'impresa a maggiori investimenti in $\mathrm{R} \& \mathrm{D}$ e all'adozione di tecnologie con rese differite nel tempo, va considerato che un'impresa multinazionale tende a scomporre la catena del valore in diverse funzioni produttive, ognuna delle quali può essere localizzata in specifici territori sulla base di strategie resource 
o market seeking. In altri termini, la capacità di associare l'intensità fattoriale di una determinata funzione produttiva alla dotazione di risorse specifiche di un territorio, costituisce una rilevante condizione di vantaggio dei gruppi multinazionali nei confronti di imprese mono-localizzate. Un tema da analizzare è dunque in quale misura i vantaggi economici dei gruppi multinazionali vengano condivisi non solo dal Paese in cui si trova la base domestica dell'impresa, ma anche dai territori nei quali sono insediate le diverse filiali produttive. Bisogna infatti considerare che $\mathrm{i}$ vantaggi di common governance delle multinazionali sono in parte contrastati dalla minore disponibilità di conoscenze sul contesto di insediamento. Ciò riguarda informazioni cruciali per la competitività dell'impresa, come il funzionamento reale del mercato del lavoro, l'esistenza e l'affidabilità delle reti di fornitura, l'osservanza delle norme e della cultura locale, il rapporto con le istituzioni. Questo tipo di svantaggi - definiti liability of foreigness (Zaheer, 1995; Cozza, Perani and Zanfei, 2016) - ha tuttavia un risvolto interessante per i sistemi locali. Infatti, la necessità da parte delle multinazionali di superare le asimmetrie informative che esse sono costrette a pagare rispetto alle imprese locali, le induce a dotarsi di capitale umano più qualificato, mettendo inoltre in campo incentivi selettivi per ottenere maggior impegno nel personale delle filiali, in particolare nel management.

Partendo da tali considerazioni ci siamo dunque posti la domanda di ricerca sugli effetti della presenza multinazionale nei distretti veneti, con particolare riferimento ai profili professionali degli occupati. Nel prossimo paragrafo esponiamo gli elementi essenziali della ricerca, che ha impiegato dati originali sugli investimenti esteri e sui comportamenti occupazionali delle imprese distrettuali, mai considerati prima in modo congiunto a questo livello di analisi.

\section{Dati dell'analisi}

Secondo l'ultima classificazione dei distretti italiani fornita dall'Istat ( $9^{\circ}$ Censimento dell'industria e dei servizi, 2015), il Nordest presenta la maggior concentrazione di sistemi produttivi distrettuali in Italia: ne vengono individuati ben 45 , pari a quasi un terzo del totale nazionale. All'interno di questa macroregione, il Veneto ospita 28 distretti distribuiti in sei settori (tab. 1), che nel complesso occupano 1,3 milioni di addetti, il 26\% del totale degli occupati nei distretti italiani. 
Tab. 1 - Specializzazione produttiva (quota distretti industriali per settore produttivo sul totale nazionale)

\begin{tabular}{lrr}
\hline Settore produttivo & Nordest & Veneto \\
\hline Beni per la casa & 54,10 & 29,20 \\
Gioielleria, oreficeria, strumenti musicali & 25,00 & 25,00 \\
Industria meccanica & 50,00 & 31,60 \\
Industria metallurgica & 25,00 & 0,00 \\
Industria alimentare & 20,00 & 6,70 \\
Pelli, cuoio e calzature & 11,80 & 11,80 \\
Tessile e abbigliamento & 18,70 & 15,60 \\
Totale & 31,91 & 19,9 \\
\hline
\end{tabular}

Fonte: elab. degli Autori su dati Istat

La classificazione Istat ha permesso di individuare le aree distrettuali presenti in Veneto e di selezionare le imprese che operano all'interno dei distretti manifatturieri della regione. Al fine di confrontare la composizione occupazionale delle imprese distrettuali a controllo italiano (Uni) con quello delle affiliate italiane delle imprese multinazionali estere (Imne) localizzate nei 28 distretti veneti, sono state utilizzati le seguenti tre banche dati, indicate di seguito ${ }^{1}$.

i) Reprint-Ice. La banca dati Reprint censisce le partecipazioni di imprese italiane all'estero ed estere in Italia, misurandone la numerosità, la consistenza economica, gli orientamenti geografici e settoriali. L'indagine riguarda, oltre ai diversi settori dell'industria manifatturiera, le utilities, le costruzioni, il commercio, le comunicazioni e gli altri servizi professionali. L'utilizzo di questo database ha consentito la raccolta di dati sulle Imne manifatturiere in Italia nel periodo 2010- 2014.

ii) Aida-Bureau van Dijk. La fonte Aida ha fornito i dati di bilancio delle imprese distrettuali, sia Uni che Imne, dal 2010 al 2013.

iii) Sistema Informativo Lavoro Veneto (Silv). La composizione occupazionale delle imprese proviene dalla banca dati Veneto Lavoro, fonte amministrativa che registra l'universo delle comunicazioni obbligatorie e riguardanti i flussi del lavoro dipendente e le forme contrattuali assimilate (come età, cittadinanza, profilo professionale, titolo di studio, ecc.).

1 Si ringraziano Bruno Anastasia, Maurizio Gambuzza e Maurizio Resera di Veneto Lavoro che hanno fornito i dati sulla composizione occupazionale delle imprese venete e ci hanno supportato nell'utilizzo della base informativa. Siamo inoltre grati a Marco Mutinelli per aver messo a disposizione i dati Reprint sulla presenza multinazionale in Italia. Ringraziamo infine Haoying Xue per l'elaborazione della mappa. 
Secondo le stime Reprint, 257 Imn estere hanno investito in Veneto, per un totale di 299 affiliate manifatturiere. Tali affiliate rappresentano l'11\% del totale delle affiliate straniere in Italia, localizzate prevalentemente in aree distrettuali (fig. 1).

Fig. 1 - Localizzazione delle multinazionali estere all'interno dei distretti veneti

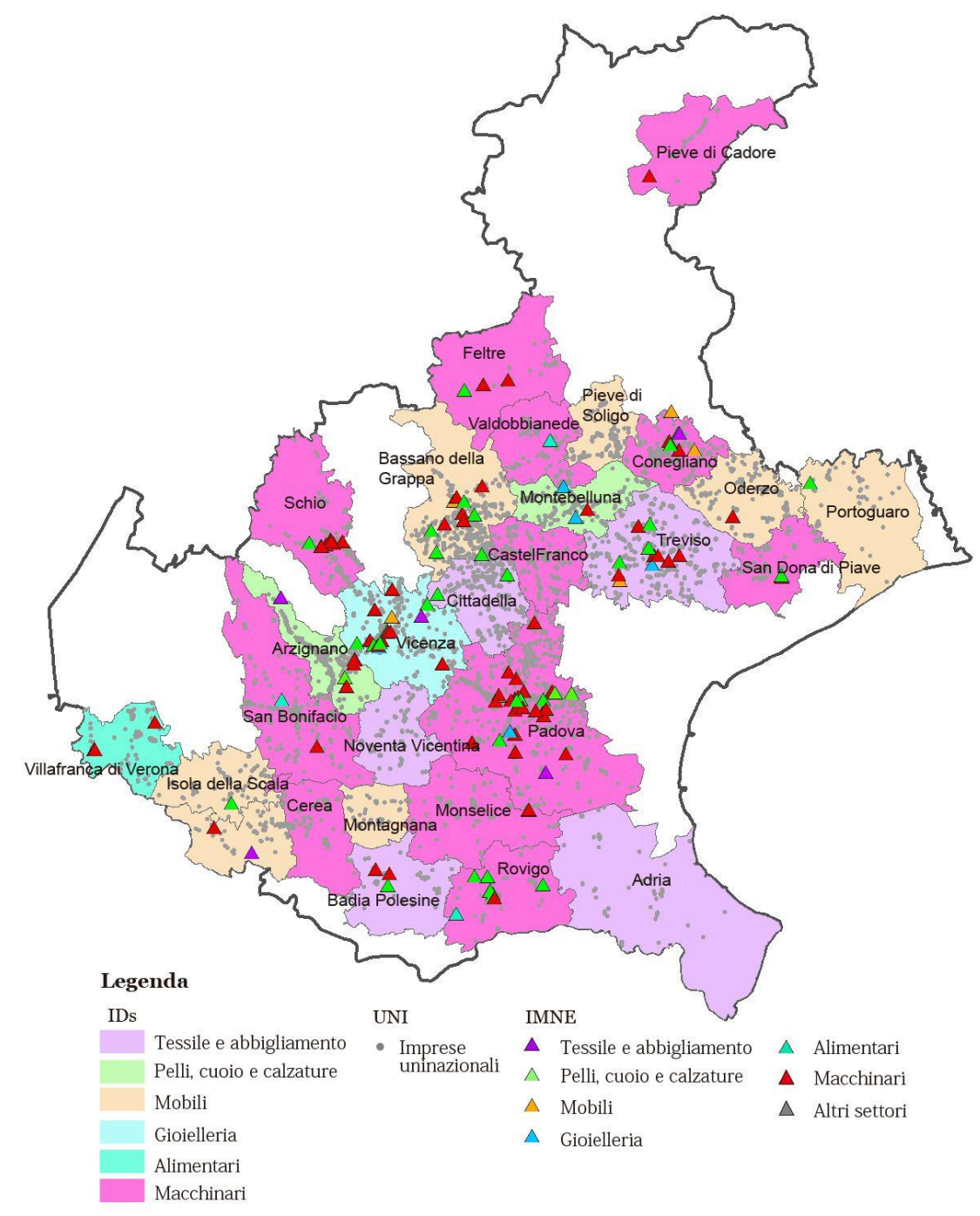

Fonte: elab. degli Autori su dati Istat e Reprint 
Il dataset finale a disposizione per l'analisi comprende 6.953 imprese distrettuali, di cui 131 Imne e 6.822 Uni che - secondo la fonte Reprint non hanno né investimenti diretti esteri (Ide), né sono state acquisite da Imn estere nel periodo di riferimento 2007-2014.

\section{Un'analisi sui comportamenti occupazionali delle imprese: multinazionali e industria locale a confronto}

Per confrontare il comportamento occupazionale delle due tipologie di imprese, è stata svolta un'analisi controfattuale sulla base delle caratteristiche aziendali delle imprese all'anno 2013 e della composizione della forza lavoro al 2014 (Mariotti and Barzotto, 2017). Al fine di costruire un appropriato gruppo di controllo di imprese uninazionali è stato utilizzato un propensity score ( $p$-score) matching, che consiste nell'elaborare un modello a scelta discreta e calcolare le differenze in termini di profili occupazionali e di caratteristiche di perfomance aziendali in base al diverso assetto proprietario. L'abbinamento dei due gruppi di imprese (Uni e Imne) è stato creato sulla base di due variabili di controllo: dimensione aziendale (espressa come logaritmo naturale del fatturato al 20102) e i seguenti tre macro settori produttivi ottenuti dalla rielaborazione della classificazione settoriale di Pavitt (1984)

i) Made in Italy diretto (quali tessile, abbigliamento e pelli) costituito da imprese di settori manifatturieri tradizionali, in cui l'innovazione si origina spesso esternamente all'impresa, ovvero da fornitori di macchine, produttori di beni intermedi, servizi tecnologici e di design.

ii) Made in Italy indiretto, imprese specializzate nel produrre macchine e tecnologie utilizzate da altre imprese del Made in Italy, con elevate conoscenze e competenze di meccanica strumentale.

iii) Altri settori, tra cui scale intensive (imprese di maggiori dimensioni, in cui l'innovazione si origina sia internamente che esternamente) e sciencebased (imprese basate su tecnologie avanzate, in cui le innovazioni derivano principalmente dai risultati di $R \& D$, come il settore farmaceutico) ${ }^{3}$.

2 La scelta di considerare il fatturato al 2010 è stata adottata al fine di controllare per il cherry-picking effect, ovvero che le imprese multinazionali estere non abbiano acquisito imprese locali più performanti (si rimanda a: Crinò and Onida, 2007; Crinò, 2010).

3 Classificazione settoriale (Nace, 2 digit): (i) Made in Italy indiretto Nace sectors: 1011 121314151617182223 31; (ii) Made in Italy diretto Nace sectors: 2526272829 30; (iii) Altri settori Nace (scale-intensive and science-based): 202124192432. 
La costruzione del controfattuale tramite Att ${ }^{4}$ prevede la stima di un modello logit, in cui la variabile dicotomica (corrispondente a 1 se l'impresa è una Imne, 0 se è un'impresa Uni) viene analizzata in base a dimensione delle imprese e settore produttivo. Tale metodo di matching è stato scelto sulla base della bontà del fit del modello statistico. Il campione finale risultante dall'analisi controfattuale consiste in 173 Imne e $637 \mathrm{Uni}^{5}$.

Lo studio mette in evidenza come le affiliate estere siano più grandi in termini di fatturato - rispetto alle imprese uni-nazionali e operino prevalentemente nei settori ad alta intensità tecnologica (tab. 2). Tale risultato conferma la tradizionale specializzazione produttiva delle imprese Uni in settori Made in Italy diretto, e di una specializzazione da parte delle Imne in settori tecnologicamente più avanzati (Made in Italy indiretto, scale intensive e science-based).

Tab. 2 - Modello logit

\begin{tabular}{ll}
\hline Variabile & Coeff. \\
\hline Fatturato 2010 $(\ln )$ & $0.9943 * * *$ \\
Made in Italy indiretto & $0.8768^{* * *}$ \\
Altri settori & $0.9837 * * *$ \\
Constante & $-12.9818^{* * *}$ \\
Numero di osservazioni & 5,729 \\
Prob > chi2 & 0.0000 \\
Pseudo R2 & 0.2084 \\
Log likelihood & -445.9296 \\
\hline
\end{tabular}

Nota: *, **, *** sono statisticamente significativi allo $10 \%, 5 \%$ e $1 \%$, rispettivamente.

Fonte: elab. degli Autori su dati Aida, Reprint, Veneto Lavoro

L'analisi controfattuale (tab. 3) riporta il maggiore e significativo impiego da parte delle affiliate estere di personale qualificato. Tali imprese pagano, in media, salari più alti per attirare i migliori lavoratori e risolvere il problema dell'asimmetria informativa, poiché spesso scontano una minore conoscenza dei mercati del lavoro locali (liability of foreigness). Non emergono invece differenze statisticamente significative in termini di produttività, né di redditività operativa. Questo risultato può essere in parte

4 L'Att (Average Treatment on Treated) è stato elaborato sulla base del 5-nearest neighbour matching method con replacement e caliper $(=0.01)$, condizionatamente al common support. Ogni azienda multinazionale straniera è accoppia a ciascun Treated con i controlli che cadono in un'intorno del propensity score predefinito. Quanto più piccolo è l'intorno (caliper) tanto migliore sarà la qualità dell'accoppiamento. .

5 Il dettaglio dei risultati può essere reso disponibile su richiesta agli autori. 
condizionato dalle strategie di transfer pricing praticate dai gruppi multinazionali per ragioni fiscali. Ma è anche la conferma di un incentivo per le imprese a controllo estero a condividere maggiormente il valore creato con il capitale umano locale.

Tab. 3 - Stima Att

\begin{tabular}{lccrrrr}
\hline Variable & Year & NATs & FMNEs & Att & Std. Err. & Sign. \\
\hline $\begin{array}{l}\text { Quota di lavoratori } \\
\text { altamente qualificati }\end{array}$ & 2014 & 4858 & 86 & 0.057 & 0.021 & Sig. \\
Valore aggiunto & 2013 & 4858 & 86 & 1.45 & 4.15 & Non Sig. \\
$\begin{array}{l}\text { Roi } \\
\begin{array}{l}\text { Costo del lavoro } \\
\text { per dipendente }\end{array}\end{array}$ & 2013 & 4858 & 86 & 37.55 & 37.62 & Non Sig. \\
\hline
\end{tabular}

Fonte: elab. degli Autori su dati Aida, Reprint, Veneto Lavoro

L'analisi degli effetti diretti delle Imne nei distretti del Veneto evidenzia quindi importanti benefici sul contesto locale, in particolare il maggior impiego lavoratori qualificati rispetto alle imprese locali.

\section{Conclusioni}

Per quanto guardata spesso con sospetto, la presenza multinazionale costituisce un importante fattore di modernizzazione anche per le economie distrettuali. Alla luce dell'analisi riassunta nel paragrafo precedente, si potrebbe anzi sostenere che gli investimenti esteri hanno in realtà contribuito a rafforzare, più che indebolire, i beni comuni industriali sui quali si fondano le qualità manifatturiere del Made in Italy dei distretti. Le multinazionali a controllo estero tendono infatti a concentrarsi in settori diversi da quelli di specializzazione dell'industria italiana, contribuendo a sviluppare una complementarità produttiva e ad arricchire la varietà e la complessità dell'economia locale. Soprattutto, le multinazionali estere forniscono maggiori sbocchi professionali alle figure più qualificate, riducendo per contro la domanda di lavoratori stranieri per le mansioni unskilled. I risultati sono statisticamente significativi sia a livello aziendale, sia prendendo in esame il sistema produttivo locale, dove la presenza multinazionale si associa anche a una maggiore intensità innovativa e a una maggiore qualità istituzionale (Corò et Al., 2016).

Il problema, semmai, è che la presenza multinazionale risulta in Italia, e anche in Veneto, ancora limitata. Inoltre, come hanno documentato recenti ricerche (Cozza e Zanfei, 2016), si sta riducendo la propensione delle imprese a controllo estero a investire su alcune funzioni strategiche, come la 
come la ricerca e lo sviluppo tecnologico in cooperazione con imprese e istituzioni la ricerca e lo sviluppo tecnologico in cooperazione con imprese e istituzioni locali.

Le ragioni della scarsa attrattività italiana per gli investimenti diretti esteri sono abbastanza note: dalla frammentazione amministrativa al cattivo funzionamento della giustizia, dalla vischiosità degli assetti proprietari delle imprese al rischio, oltre una soglia dimensionale, del prevalere di relazioni industriali conflittuali. Anche la presenza di infrastrutture moderne ha un peso nelle strategie di investimento estero, così come l'esistenza di spazi metropolitani ben organizzati, in grado di fornire servizi qualificati e, più in generale, un ambiente attrattivo per manager e tecnici internazionali. Per i distretti veneti questi problemi non possono essere affrontati internamente, ma richiedono una politica comune che sembra tuttavia ancora piuttosto lontana dall'orizzonte dei decisori pubblici regionali. Anche perché, alle difficoltà strutturali, si aggiunge un atteggiamento culturale che risulta spesso scettico, se non ostile, alla presenza estera sull'economia locale. Modificare questo atteggiamento è dunque un passaggio fondamentale per organizzare un'efficace politica di attrazione degli investimenti esteri come parte di una più generale politica per lo sviluppo industriale e la qualità dell'occupazione.

\section{Riferimenti bibliografici}

Barzotto M., Corò G. and Volpe M. (2016). Territorial capital as a company intangible: Exploratory evidence from ten Italian multinational corporations. Journal of Intellectual Capital, 17(1): 148- 167, doi: 10.1108/JIC-09-2015-0077.

Buciuni G. and Pisano G.P. (2015). Can Marshall's Clusters Survive Globalization?, Harvard Business School Working Paper, No. 15-088, <www.hbs.edu/ faculty/Publication\%20Files/15-088_cd4f1da8-4988-408b-b7eb8c0e5798a39 8.pdf>.

Castellani D. and Zanfei A. (2006). Multinational firms, Innovation and productivity. Northampton (MA): Edward Elgar.

Crinò R. (2010). Employment Effects of Service Offshoring: Evidence from Matched Firms. Economic Letters, 107(2): 253-256, doi: doi.org/10.1016/ j.econlet.2010.01.039.

Crinò R. and Onida F. (2007). Foreign Ownership and Economic Performance in Italy: Not all is Cherry-Picking!. Milan: Bocconi University CESPRI WP 207, November, <www.etsg.org/ETSG2007/papers/onida.pdf>.

Corò G., Costantini M., Garalova P., Iapadre L. e Mutinelli M. (2016). Rapporto CER Integrazione internazionale e occupazione. Roma: CER - Centro Europa Ricerche, vol. 4.

Corò G. e Micelli S. (2006). I Nuovi Distretti Produttivi. Innovazione, internazionalizzazione e competitività dei territori. Venezia: Marsilio. 
Corò G. e Volpe M. (2003). Frammentazione produttiva e apertura internazionale nei sistemi di piccola e media impresa. economia e società regionale, 1: 67-107.

Cozza C., Perani G. and Zanfei A. (2016). Are multinationals better at creating technical linkages with local firms and institutions? Lund University: Papers in Innovation Studies, Circle, 2016/14, <http://swopec.hhs.se/lucirc/abs/lucirc 2016_014.htm>.

Cozza C. e Zanfei A. (2016). Multinazionali e creazione di legami con imprese e università in Italia. In: Rapporto ICE 2015-2016. L'Italia nell'economia internazionale: 297-304, <www.ice.it/statistiche/archivio_rapporti/2016/Rapporto \%20Ice\%20-\%202016.pdf $>$.

De Marchi V. and Grandinetti R. (2014). Industrial districts and the collapse of the Marshallian model: Looking at the Italian experience. Competition and Change, 18(1): 70-87, doi: 10.1179/1024529413Z.00000000049.

Dunning J.H. (1993). Multinational enterprises and the global economy. Wokingham: Addison-Wesley.

Giunta A. e Rossi S. (2017). Cosa sa fare l'Italia. La nostra economia dopo la grande crisi. Bari: Laterza.

Guelpa F. e Micelli S., a cura di (2007). I distretti industriali del terzo millennio. Dalle economie di agglomerazione alle strategie d'impresa. Bologna: il Mulino.

Istat (2015). $9^{\circ}$ Censimento dell'industria e dei servizi e Censimento delle istituzioni non profit. I distretti industriali 2011. Roma: Istat.

Mariotti I. and Barzotto M. (2017). Inward FDI and Skilled Labour Force in Veneto's Industrial Districts. In: Hervas Oliver L.J. and Belussi F., eds. Cluster Advantage and Firm Performance. Frankfurt: Springer Verlag.

Morrison A. (2008). Gatekeepers of knowledge within industrial districts: Who they are, how they interact. Regional Studies, 42(6): 817-835, doi: 10.1080/00343400701654178.

Pavitt K. (1984). Sectoral patterns of technical change: Towards a taxonomy and a theory. Research Policy, 13: 343-373.

Pisano G.P. and Shih W.C. (2012). Producing Prosperity. Why America Needs a Manufacturing Renaissance. Boston: Harvard Business School Press.

Rullani E. (2015). Investimenti, rischi e governance di impresa. In: Capellin R., a cura di. Investimenti, innovazione e città Una nuova politica industriale per la crescita. Milano: Egea.

Zaheer S. (1995). Overcoming the liability of foreignness. Academy of Management Journal, 38(2): 341-363. 